\title{
MAFB as a novel regulator of human adipose tissue inflammation
}

\author{
Annie M. L. Pettersson ${ }^{1}$ • Juan R. Acosta ${ }^{1}$. Christel Björk ${ }^{1}$ • Johan Krätzel ${ }^{1}$ • \\ Britta Stenson $^{1}$ - Lennart Blomqvist ${ }^{1}$ - Nathalie Viguerie ${ }^{2}$ - Dominique Langin ${ }^{2,3}$. \\ Peter Arner $^{1}$ - Jurga Laurencikiene ${ }^{1}$
}

Received: 2 March 2015 / Accepted: 4 June 2015 / Published online: 27 June 2015

(C) Springer-Verlag Berlin Heidelberg 2015

\begin{abstract}
Aims/hypothesis Dysregulated expression of metabolic and inflammatory genes is a prominent consequence of obesity causing insulin resistance and type 2 diabetes. Finding causative factors is essential to understanding progression of these pathologies and discovering new therapeutic targets. The transcription factor V-maf musculoaponeurotic fibrosarcoma oncogene homologue B (MAFB) is highly expressed in human white adipose tissue (WAT). However, its role in the regulation of WAT function is elusive. We aimed to characterise MAFB expression and function in human WAT in the context of obesity and insulin resistance.

Methods MAFB mRNA expression was evaluated in human WAT from seven cohorts with large inter-individual variation in BMI and metabolic features. Insulin-induced adipocyte lipogenesis and lipolysis were measured and correlated with $M A F B$ expression. $M A F B$ regulation during adipogenesis and the effects of $M A F B$ suppression in human adipocytes
\end{abstract}

Annie M.L. Pettersson and Juan R. Acosta contributed equally to this study.

Electronic supplementary material The online version of this article (doi:10.1007/s00125-015-3673-x) contains peer-reviewed but unedited supplementary material, which is available to authorised users.

Jurga Laurencikiene

Jurga.Laurencikiene@ki.se

Department of Medicine Huddinge, Lipid laboratory, Karolinska Institutet, Novum, NVS D4, Hälsovägen 7,

14186 Stockholm, Sweden

2 Inserm UMR1048, Institute of Metabolic and Cardiovascular Diseases, Obesity Research Laboratory, and Paul Sabatier University, Toulouse, France

3 Franco-Czech Laboratory for Clinical Research on Obesity, Third Faculty of Medicine, Prague, Czech Republic was investigated. $M A F B$ regulation by TNF- $\alpha$ was examined in human primary adipocytes and THP-1 monocytes/ macrophages.

Results MAFB expression in human adipocytes is upregulated during adipogenesis, increases with BMI in WAT, correlates with adverse metabolic features and is decreased after weight loss. $M A F B$ downregulation decreases proinflammatory gene expression in adipocytes and interferes with TNF- $\alpha$ effects. Interestingly, MAFB is differentially regulated by TNF- $\alpha$ in adipocytes (suppressed) and THP-1 cells (upregulated). Further, MAFB is primarily expressed in WAT macrophages/ monocytes and its expression correlates with macrophage and inflammatory markers.

Conclusions/interpretation Our findings indicate that MAFB is a regulator and a marker of adipose tissue inflammation, a process that subsequently causes insulin resistance.

Keywords Adipocytes · Adipose tissue - Inflammation · Insulin resistance $\cdot$ MAFB $\cdot$ Metabolic syndrome

$\begin{array}{ll}\text { Abbreviations } \\ \text { C/EBP } \delta & \text { CCAAT/enhancer binding protein } \delta \\ \text { hMSCs } & \text { Human mesenchymal stem cells } \\ \text { JNK } & \text { c-Jun N-terminal kinase } \\ \text { MAFB } & \text { V-maf musculoaponeurotic fibrosarcoma } \\ & \text { oncogene homologue B } \\ \text { MCP-1 } & \text { Monocyte chemoattractant protein 1 } \\ \text { qRT-PCR } & \text { Quantitative RT-PCR } \\ \text { SAM } & \text { Significance analysis of microarrays } \\ \text { scWAT } & \text { Subcutaneous WAT } \\ \text { SVF } & \text { Stromal vascular fraction } \\ \text { shRNA } & \text { Short hairpin RNA } \\ \text { siRNA } & \text { Small interfering RNA }\end{array}$


TG Triacylglycerol

WAT White adipose tissue

\section{Introduction}

White adipose tissue (WAT) is composed of various cell types, including adipocytes and macrophages. During the development of obesity, maladaptive modifications of WAT metabolism and inflammation occur due to dysregulated expression of key regulatory intracellular proteins. In turn, this alters the main functions of the adipose tissue, which can lead to development of the metabolic syndrome and detrimental effects on human health and longevity [1].

An important factor regulating changes in metabolism during WAT expansion is an increase in secretion of proinflammatory proteins. An elevated release of monocyte chemoattractant protein 1 (MCP-1; encoded by CCL2) from adipocytes promotes macrophage infiltration of adipose tissue, which further consolidates the inflamed state by releasing a number of proinflammatory peptides [2]. Being a significant constituent of WAT, the macrophage population has gained increased attention as an important modulator of obesitycaused inflammation [3]. However, some factors are produced by both adipocytes and macrophages, including potent proinflammatory cytokines such as MCP-1, IL-6 and TNF- $\alpha$ [4-6], which all promote insulin desensitisation and development of type 2 diabetes [7].

V-maf musculoaponeurotic fibrosarcoma oncogene homologue B (MAFB) is a widely expressed basic leucine zipper transcription factor belonging to the Maf family [8]. Discovered as an oncogene [9], it has later been designated as a regulator of hindbrain segmentation [10], blood cell development [11, 12], and osteoclast [13], beta cell [14] and macrophage differentiation [15]. Thus, MAFB has been implicated in developmental and differentiation processes. Moreover, MAFB is a transcriptional activator of insulin and glucagon expression in pancreatic islets $[16,17]$, linking its function to metabolism and development of diabetes [18].

In a human monocyte cell line, $M A F B$ is upregulated by TNF- $\alpha$ treatment, and in turn activates transcription of CCL2, implying a possible role in inflammation [19]. In keratinocytes, $M A F B$ expression is regulated by CCAAT/enhancer binding protein $\delta(\mathrm{C} / \mathrm{EBP} \delta)$, a transcription factor involved in early induction of adipogenesis [20]. These data suggest that MAFB may also be of significance for adipose tissue biology. Yet, this transcription factor has not previously been implicated in WAT function in obesity or insulin resistance. In the present study, we aimed to characterise the role of MAFB in human adipocytes and adipose tissue.

\section{Methods}

Clinical cohorts Seven different cohorts of individuals were used to examine MAFB expression in human WAT. The cohorts are described in the electronic supplementary material (ESM) Methods.

Cell cultures and treatments In this study, two different human adipocyte in vitro cell cultures were used; primary stromal vascular fraction (SVF)-derived pre-adipocytes and human mesenchymal stem cells (hMSCs). The differentiation protocols and cell culture treatments are specified in the ESM Methods.

Fat cell lipolysis and lipogenesis Lipolysis and lipogenesis procedures are described in the ESM Methods.

mRNA and miRNA analysis Total RNA was extracted and gene expression was quantified as described [21] and in the ESM Methods. Quantification of mRNA expression was performed using the comparative $C_{t}$ method $\left(2^{\Delta \mathrm{C}_{\mathrm{t}}-\text { target gene }} / 2^{\Delta \mathrm{C}_{\mathrm{t}}-\text { reference gene }}\right)$. SYBR Green primer sequences are listed in ESM Table 1.

Western blot Cell lysis and western blot for protein expression analysis was performed as previously described [22]. The following antibodies were used: anti-MAFB (GenWay, San Diego, CA, USA), secondary anti-rabbit IgG antibody (Sigma Aldrich, St Louis, MO, USA), anti- $\beta$-actin (Sigma Aldrich) and anti- $\alpha$-tubulin (Cell Signaling Technology, Danvers, MA, USA).

ELISA Secreted IL-6 and MCP-1 were measured in conditioned medium from hMSC-derived adipocytes using kits from R\&D Systems (Bio-Techne, Abingdon, UK). TNF- $\alpha$ release from abdominal subcutaneous WAT (scWAT) was determined as described [23]. In short, WAT explants were incubated for $2 \mathrm{~h}$ after which TNF- $\alpha$ secretion into medium was quantified with ELISA (R\&D Systems, Minneapolis, MN, USA) as ng per $10^{7}$ adipocytes. The number of cells in the explant was estimated based on triacylglycerol (TG) content and the average cell volume.

Neutral lipid and DNA staining At day 13 of differentiation, hMSC-derived adipocytes were washed with PBS and fixed with $4 \%$ paraformaldehyde solution (PFA) containing $0.123 \mathrm{~mol} / 1 \mathrm{NaH}_{2} \mathrm{PO}_{4} \times \mathrm{XH}_{2} \mathrm{O}, 0.1 \mathrm{~mol} / \mathrm{l} \mathrm{NaOH}$ and $0.03 \mathrm{~mol} / \mathrm{l}$ glucose for $10 \mathrm{~min}$ at room temperature. Fixed cells were washed with PBS and stained with Bodipy 493/503 $(0.2 \mu \mathrm{g} / \mathrm{ml}$ PBS; Molecular probes, Life Technologies, Carlsbad, CA, USA) and Hoechst $33342(2 \mu \mathrm{g} / \mathrm{ml} \mathrm{PBS}$, Molecular probes) for $20 \mathrm{~min}$ at room temperature. After washing with PBS, accumulation of intracellular lipids 
(Bodipy staining) and cell number (Hoechst staining for nuclei) were quantified with Acumen $\mathrm{XX} 3$ imager (TTP Labtech, Hertfordshire, UK). Lipid accumulation was normalised by cell number.

RNA interference RNA interference is described in detail in the ESM Methods.

SVF sorting by flow cytometry scWAT SVF was sorted by flow cytometry as described [21]. CD45+/CD14- cells represented the fraction of leucocytes and CD45+/CD14+ cells corresponded to the macrophage/monocyte fraction. Cell purity was investigated after each sorting and corresponded to $95.1 \pm 2.9$ for the monocyte/macrophage fraction and $98.8 \pm 0.87$ for the leucocyte fraction. Expression of $M A F B$ was analysed by quantitative (q)RT-PCR in mature adipocytes $(n=11)$, leucocytes $(n=11)$ and monocytes/macrophages $(n=12)$.

Predicting genes binding to the $M A F B$ promoter and mediating TNF $\alpha$ signalling Prediction and selection of the genes that could possibly regulate the $M A F B$ promoter are described in the ESM Methods.

Statistics Student's $t$ test as well as non-parametric MannWhitney and Wilcoxon tests were used for statistical analysis. Non-parametric Kruskal-Wallis, Dunn's (post hoc) tests as well as significance analysis of microarrays (SAM) were used for multiple comparisons. Data are presented as mean $\pm \mathrm{SD}$. Correlations between gene expression, BMI and clinical variables were analysed by linear regression; in addition, Pearson or Spearman correlation analysis was performed. The level of significance was set to $0.05: * p<0.05 ; * * p<0.01$; $* * * p<0.001$. Multiple regression analysis was used to adjust for multiple variables. Values obtained in clinical patient groups were logarithmically $\left(\log _{10}\right)$ transformed for normal distribution where indicated.

\section{Results}

$M A F B$ expression in human scWAT is regulated by BMI and metabolic status Microarray profiling of gene expression in scWAT obtained from cohort 1 revealed MAFB as an obesity-regulated transcription factor. $M A B F$ was correlating positively and significantly with BMI (Fig. 1a). This finding was confirmed on protein level using western blots of scWAT from 17 non-obese and 16 obese individuals (Fig. 1b). In cohort 2, MAFB expression was increased in obese patients with the metabolic syndrome compared with obese healthy individuals, with mean BMI comparable in the two groups (Fig. 1c). In this cohort $M A F B$ was also correlating with $\mathrm{BMI}$ and features of the metabolic syndrome, where these

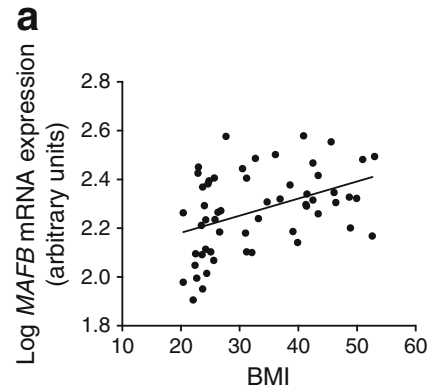

b
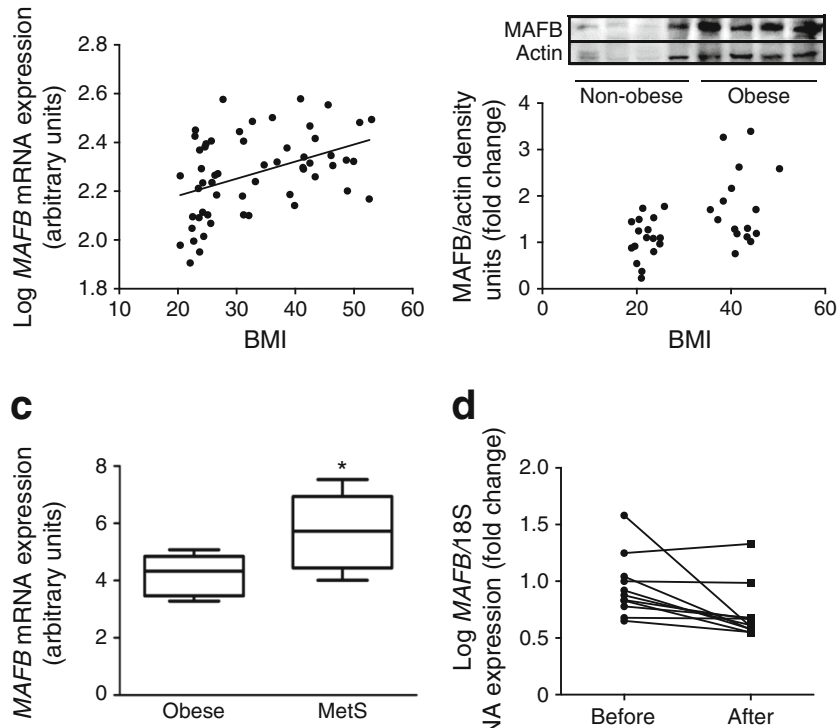

d

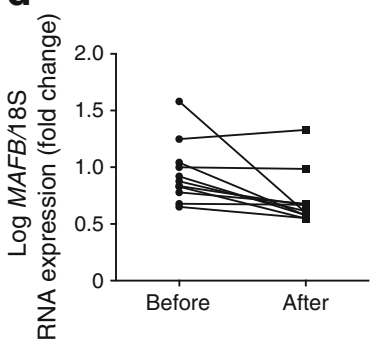

Fig. $1 M A F B$ expression is regulated by adipose tissue mass in humans. (a) Linear regression of $M A F B$ mRNA expression (arbitrary units of fluorescence intensity [microarray]) and BMI in scWAT in cohort 1 (Pearson $r=0.4108, p=0.0013$ ). (b) MAFB protein expression in scWAT from non-obese and obese individuals is shown in a representative western blot. Relations between relative protein expression and BMI in 17 nonobese and 16 obese individuals were analysed (Spearman $\rho=0.4333, p=$ 0.018). (c) Expression of $M A F B$ mRNA in scWAT was assessed by microarray analysis and compared in obese vs obese with the metabolic syndrome (MetS) ( $n=8$ in each category). The Mann-Whitney test was performed to compare the groups. (d) MAFB mRNA expression was quantified in scWAT after weight loss by qRT-PCR $(n=11)$. Paired samples are interconnected. The Wilcoxon test was performed for group comparison $(p=0.0049) .{ }^{*} p<0.05$

two variables could explain approximately $60 \%$ of variation of $M A F B$ expression (Table 1). In line with these data, we found that $M A F B$ expression in scWAT obtained from cohort 3 was reduced after weight loss (Fig. 1d). This trend was further established in cohort 4, where $M A F B$ expression in scWAT was significantly diminished after a very low calorie $\operatorname{diet}(\mathrm{VLCD})$ intervention programme for weight loss and subsequent weight maintenance (ESM Table 2). MAFB was ranked as 166 among 667 intervention-regulated genes using SAM ( $q$ value $0 \%)$.

$M A F B$ expression correlates negatively with lipogenesis and lipolysis in human adipocytes To determine the relation

Table 1 Relation of $M A F B$ mRNA expression with BMI and the metabolic syndrome, analysed by multiple regression

\begin{tabular}{lllllll}
\hline Explanatory variable & \multicolumn{2}{l}{ Single regression } & & \multicolumn{2}{l}{ Multiple regression } \\
\cline { 2 - 3 } \cline { 5 - 6 } & $r$ & $p$ & & Partial $r$ & Partial $p$ \\
\hline BMI & 0.670 & $<0.0001$ & & 0.608 & $<0.0001$ \\
Metabolic syndrome & 0.608 & $<0.0001$ & 0.529 & 0.002 \\
\hline
\end{tabular}


of $M A F B$ expression to adipocyte metabolism, insulinstimulated lipogenesis and isoprenaline-stimulated lipolysis were determined in isolated mature adipocytes from 56 human participants from cohort 1 with a broad range of BMIs (20.451.0). $M A F B$ expression correlated negatively with adipocyte responsiveness to insulin in induction of lipogenesis (Fig. 2a) and responsiveness to isoprenaline in lipolysis measurements (Fig. 2b). These correlations remained statistically significant after correction for variations in BMI $(p=0.014$ and $p=$ 0.000017 , respectively).

MAFB is induced by adipogenesis and is required for TNF- $\alpha$-mediated effects on lipid accumulation and lipolysis To further investigate MAFB function, we quantified $M A F B$ expression during in vitro adipogenesis of hMSC-derived adipocytes and found that $M A F B$ was strongly upregulated (Fig. 3a). To assess the importance of MAFB for the differentiation and metabolic features of the adipocyte, we performed small interfering RNA (siRNA)-mediated knockdown in hMSC-derived adipocytes. The efficiency of knockdowns was $75 \%$ on the mRNA level (ESM Fig. 1a) and $50 \%$ on protein level (ESM Fig. 1). Neither early (day 1 of differentiation) nor late (day 9 of differentiation) knockdown of
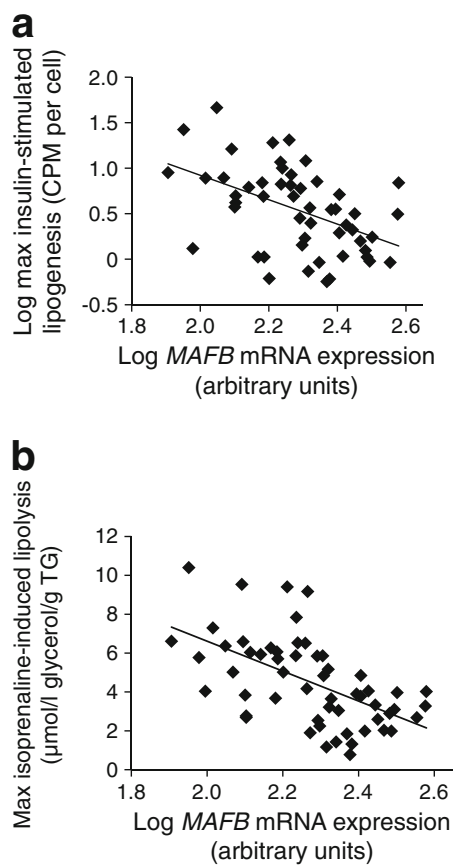

Fig. 2 Negative associations of MAFB expression (arbitrary units of fluorescence intensity [microarray]) with stimulated lipolysis and lipogenesis in mature adipocytes. Insulin-stimulated lipogenesis (a) (Pearson $r=0.4747, p=0.0004$ ) and isoprenaline-induced lipolysis (Pearson $r=0.5656, p<0.0001$ ) (b) were measured in adipocytes from 56 individuals. Correlations with $M A F B$ mRNA expression were investigated by regression analysis. The rate of lipolysis was quantified as release of glycerol per g TG. Lipogenesis was measured as tritium-labelled glucose incorporation into lipids and expressed as cpm per cell. Values were logarithmically $\left(\log _{10}\right)$ transformed for normal distribution. Max, maximum
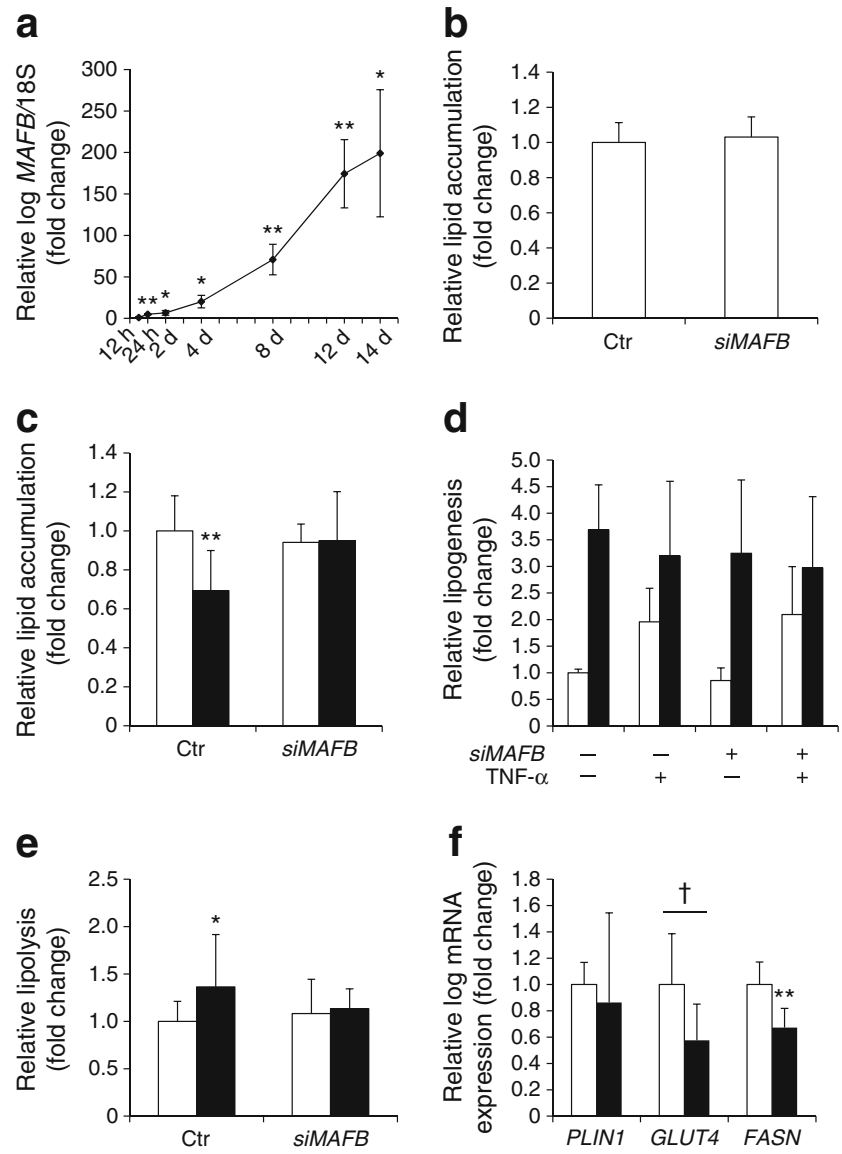

Fig. $3 M A F B$ expression is induced by differentiation of hMSC-derived adipocytes and reverses TNF- $\alpha$-mediated effects on lipid accumulation and lipolysis. (a) $M A F B$ expression was quantified between $0 \mathrm{~h}$ and 14 days (d) of in vitro differentiation of adipose-derived hMSCs $(n=3)$. Quantification was normalised to expression at $12 \mathrm{~h}$ because $M A F B$ was not detected at earlier time points of differentiation. (b, c) Lipid accumulation was assessed by neutral lipid staining and quantification at full differentiation state. SiRNA was delivered on the day before induction of differentiation $(n=2)(\mathbf{b})$, or $72 \mathrm{~h}$ before full differentiation $(n=3)(\mathbf{c})$ of hMSC-derived adipocytes treated with TNF- $\alpha$ (black bars) or without (white bars). Accumulation was normalised to non-targeting control (Ctr). (d) Lipogenesis in hMSC-derived adipocytes treated with nontargeting control or $S i M A F B$ with or without TNF- $\alpha$ stimulation was analysed by uptake of D-[3- $\left.{ }^{3} \mathrm{H}\right]$ glucose and normalised to protein concentration $(n=3)$. Basal (white bars) and insulin-stimulated glucose uptake (black bars) was measured. Lipogenesis was normalised to the basal condition in non-targeting siRNA-treated cells. (e) Lipolysis was assessed in hMSC-derived adipocytes treated with non-targeting control siRNA or siMAFB, and with (black bars) or without (white bars) TNF- $\alpha(n=3)$. Glycerol release was normalised to non-targeting control. (f) Expression (qRT-PCR) of PLIN1, SLC2A4 (GLUT4) and FASN in hMSC-derived adipocytes treated with non-targeting control siRNA (white bars) or siMAFB (black bars) in combination with TNF- $\alpha$. Expression was normalised to the TNF- $\alpha$ effect in non-targeting control-treated samples for each gene. ${ }^{*} p<0.05 ;{ }^{* *} p<0.01 ;{ }^{\dagger} p=0.053$

MAFB affected the level of adipogenesis/lipid accumulation in in vitro differentiated adipocytes (Fig. $3 \mathrm{~b}$ and 3c, respectively). On the other hand, MAFB-specific siRNA (siMAFB) treatment reversed the inhibition of lipid accumulation caused by TNF- $\alpha$ (Fig. $3 \mathrm{c}$ ). Basal and insulin- 
stimulated lipogenesis was not affected by the knockdown, whereas TNF- $\alpha$ stimulation diminished the insulin response as expected (Fig. 3d). We further investigated the effect of MAFB knockdown on lipolysis, measured by the amount of glycerol released into the conditioned medium over the final $48 \mathrm{~h}$ of differentiation. Basal lipolysis was not affected by siMAFB, whereas TNF- $\alpha$-induced glycerol release was inhibited by MAFB knockdown (Fig. 3e). To uncover a possible mechanism behind the inhibitory effects of MAFB knockdown on TNF- $\alpha$-regulated genes, we quantified mRNA expression of perilipin 1 (PLINI), fatty acid synthase $(F A S N)$ and glucose transporter 4 (SLC2A4, also known as GLUT4) after TNF- $\alpha$ stimulation in hMSC-derived adipocytes with or without simultaneous MAFB knockdown. Treatment with TNF- $\alpha$ reduced the expression of PLINI, FASN and SLC2A4 dramatically (to $1.8 \%, 20 \%$, and $1.5 \%$ of baseline expression, respectively; data not shown) as expected. MAFB knockdown did not affect basal expression levels of the three genes in itself (data not shown). However, TNF- $\alpha$ mediated suppression of $F A S N$ was markedly diminished upon MAFB knockdown (Fig. 3f).

MAFB regulates inflammation in adipocytes Based on previous findings that MAFB regulates expression of inflammatory genes in activated monocytes, we hypothesised that MAFB might also regulate the inflammatory phenotype of adipocytes. SiRNA-mediated knockdown of MAFB did not regulate basic expression of MCP-1, IL-6 or IL-8 (data not shown). When stimulating the cells with TNF- $\alpha$, a dramatic and expected increase in the expression of MCP-1, IL- 6 and IL-8 was observed $(293 \times, 319 \times$ and $18,000 \times$, respectively). When TNF- $\alpha$ stimulation was combined with $\operatorname{siMAFB}$, the induction $I L-6$ mRNA was reduced by $50 \%$ (Fig. 4a). The TNF- $\alpha$-stimulated increase in CCL2 and $I L-8$ expression was not affected. Furthermore, we measured the level of secretion of MCP-1 and IL-6 into the conditioned medium of hMSC-derived adipocytes treated with non-targeting control or $s i M A F B$, in combination with TNF- $\alpha$. TNF- $\alpha$-induced IL-6 secretion was repressed by MAFB knockdown, whereas MCP-1 remained unaltered (Fig. 4b). To investigate the effects of $S M A F B$ in another model of primary human adipocytes, we downregulated MAFB in adipocytes that were directly, without expansion, differentiated in vitro from scWAT SVF. We infected cells with adenoviral vector carrying MAFB-specific short hairpin RNA (shRNA) 2 days before full differentiation. The shRNA specifically and in a concentration-dependent manner downregulated MAFB expression (ESM Fig. 1c). In this cell model, MAFB downregulation significantly attenuated expression of $C C L 2$ and slightly, but not significantly, reduced expression of $I L-6$ (Fig. 4c).

MAFB expression is differentially regulated by TNF- $\alpha$ in adipocytes and monocytes/macrophages It has been reported that TNF- $\alpha$ induces MAFB expression in the THP-1 monocyte cell line [19]. Therefore, we aimed to investigate if $M A F B$ expression in adipocytes is also regulated by the proinflammatory environment present in obese WAT. We stimulated in vitro differentiated adipocytes with different concentrations of TNF- $\alpha$ and surprisingly observed strong and concentration-dependent $M A F B$ downregulation. In contrast, TNF- $\alpha$, at the same concentrations, upregulated MAFB expression in THP-1 monocytes (compare Fig. 5a and 5b). A similar induction was also observed in THP-1 cells differentiated to macrophages, although the amplitude was lower in macrophages compared with monocytes (data not shown). The effect of TNF- $\alpha$ in both cell types was already visible after $6 \mathrm{~h}$ of stimulation, indicating a direct influence on gene expression (Fig. 5c,d) and possibly a short half-life of $M A F B$ mRNA. To determine which kinases in the TNF- $\alpha$-signalling
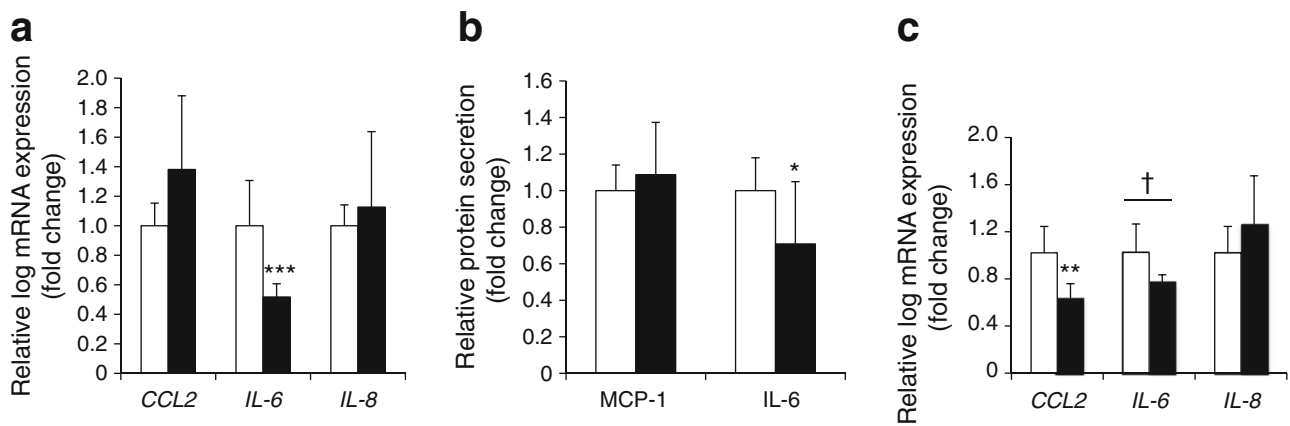

Fig. 4 MAFB regulates TNF- $\alpha$-induced IL-6 expression. (a) TNF- $\alpha$ stimulated mRNA expression of $C C L 2, I L-6$ and $I L-8$ was quantified in hMSC-derived adipocytes treated with non-targeting control (white bars) or siMAFB (black bars) $(n=3)$. Expression was normalised to the TNF- $\alpha$ effect in non-targeting control-treated samples. (b) TNF- $\alpha$-mediated protein secretion of MCP-1 and IL- 6 into conditioned medium was measured by ELISA after treatment with TNF- $\alpha$ for $48 \mathrm{~h}$ in combination with nontargeting siRNA (white bars) or siMAFB (black bars) in hMSC-derived

adipocytes $(n=3)$. Expression was normalised to TNF- $\alpha$ effect in nontargeting control-treated samples. (c) Expression of CCL2, IL-6 and $I L-8$ was quantified in primary adipocytes differentiated in vitro in control (white bars) and MAFB shRNA-treated (black bars) samples $(n=3) .100$ multiplicity of infection (MOI) was used. The expression of each gene was normalised to samples treated with non-targeting control. ${ }^{*} p<0.05$; $* * p<0.01 ; * * * p<0.001 ;{ }^{\dagger} p=0.053$ 

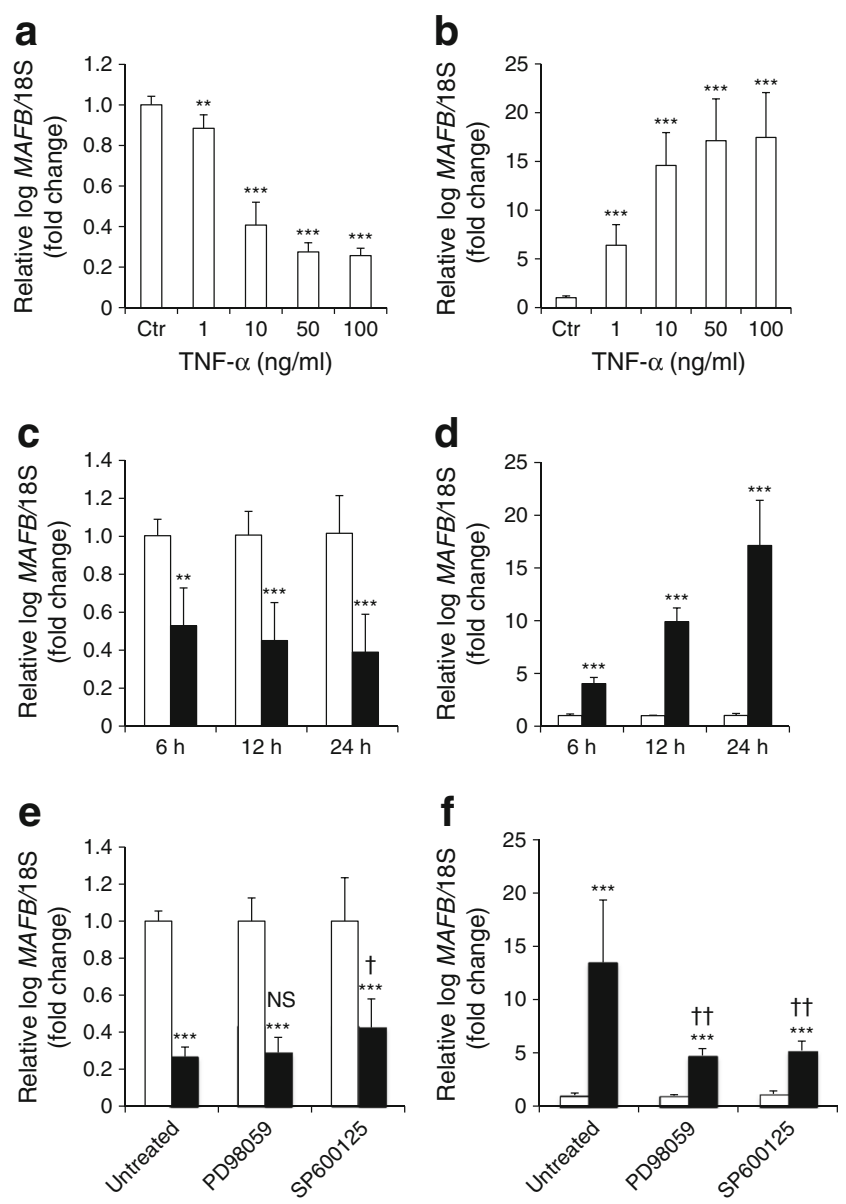

Fig. 5 TNF- $\alpha$ stimulation differentially regulates $M A F B$ expression in THP-1 cells and adipocytes. hMSC-derived adipocytes (a) and THP-1 cells (b) were treated with a range of concentrations of TNF- $\alpha$ (white bars) for $24 \mathrm{~h}$ and $M A F B$ expression was measured. Adipocytes (c) and THP-1 cells (d) were treated with DMSO (white bars) or TNF- $\alpha$ (black bars) for different durations. Adipocytes (e) and THP-1 cells (f) were treated with DMSO (white bars) or TNF- $\alpha$ for $24 \mathrm{~h}$ (black bars) with or without addition of inhibitors specific for $\mathrm{p} 44 / 42$ (PD98059) or JNK (SP600125) $(n=3) .{ }^{* *} p<0.01$ and $* * * p<0.001$ counted against unstimulated control in the same condition; ${ }^{\dagger} p<0.05$ and ${ }^{\dagger \dagger} p<0.001$ against the TNF- $\alpha$ effect in the control sample; NS, non-significant against TNF- $\alpha$ effect in the control sample

cascade mediate regulation of $M A F B$ expression, prior to TNF- $\alpha$ treatments we added inhibitors for c-Jun N-terminal kinase (JNK; SP600125), p44/42 (PD58059) and NF-kB (I229), which are key regulators of TNF- $\alpha$ signalling pathways. Only JNK inhibitor significantly attenuated TNF- $\alpha$ mediated $M A F B$ suppression in adipocytes (Fig. 5e), while both JNK and p44/42 kinase inhibitors significantly suppressed the upregulation of MAFB expression by TNF- $\alpha$ in THP-1 cells (Fig. 5f), indicating differential regulation of $M A F B$ expression in these two cell types. At suboptimal concentrations, I229 did not reverse the effects of TNF- $\alpha$ on $M A F B$ expression. Higher concentrations of I229 could not be studied due to toxic effects. $C C L 2$ and $I L-6$, known to be regulated by TNF- $\alpha$, were used as positive controls for
TNF- $\alpha$ stimulation in adipocytes, and CCL2 was used to verify the effect of inhibitor treatments (ESM Fig. 2a-d). In search for a mechanism of cell type-specific MAFB regulation by TNF- $\alpha$, we quantified the expression of miR-148a in TNF- $\alpha$-treated adipocytes and THP- 1 cells. This miRNA has been reported to target MAFB in peripheral blood mononuclear cells [24]. However, its expression was not different between cell types nor was it responsive to TNF- $\alpha$ treatment (ESM Fig. 3a). To further uncover a possible mechanism that causes cell type-specific regulation of MAFB, we used bioinformatics tools (as described in ESM Methods) to find transcription factors that could be involved. Three genes were selected (SPI1, CREB1 and MAZ). SiRNA knockdowns of these transcription factors in combination with TNF- $\alpha$ stimulation in THP-1 cells demonstrated that suppression of SPI1 diminished the impact of TNF- $\alpha$ on MAFB mRNA levels (ESM Fig. 3b), while knockdowns of $C R E B 1$ and $M A Z$ did not alter transcription (data not shown). To confirm differential expression of SPII in adipocytes vs macrophages, we performed qRT-PCR in adipocytes as well as in paired samples of scWAT macrophages and leucocytes separated by FACS. SPII was nearly absent in adipocytes but highly expressed in scWAT macrophages (ESM Fig. 3c).

$M A F B$ expression in adipose tissue macrophages is a marker of proinflammatory adipose tissue phenotype As $M A F B$ was downregulated by TNF- $\alpha$ in adipocytes, yet upregulated in THP-1 cells, we hypothesised that elevated levels of $M A F B$ expression in obesity most likely reflects macrophage infiltration and the inflammatory phenotype of WAT. To this end, we assessed $M A F B$ expression in macrophages and leucocytes obtained from scWAT SVF of human individuals in cohort 6 by flow cytometry sorting and compared with paired samples of adipocytes. Quantification of MAFB mRNA showed that it is predominantly expressed in WAT macrophages (Fig. 6a). When lean and obese participants were separated, there was no difference in $M A F B$ expression in isolated adipocytes obtained from lean and obese individuals. However, $M A F B$ expression was strongly increased in obese intact scWAT (Fig. 6b). In addition, we observed a very strong correlation of mRNA expression of $M A F B$ and macrophage markers $C D 14$ (Fig. 6c) and $C D 68\left(R^{2} 0.7614, p<0.0001\right.$; data not shown) as well as with TNF- $\alpha$ secretion rate from scWAT explants (Fig. 6d).

\section{Discussion}

MAFB is a transcription factor regulating tissue development, cell differentiation and cancer. In this study we demonstrate for the first time that $M A F B$ expression is strongly induced by adipogenesis in human adipocytes and that MAFB mediates TNF- $\alpha$-induced expression of IL- 6 in hMSC-derived 
a
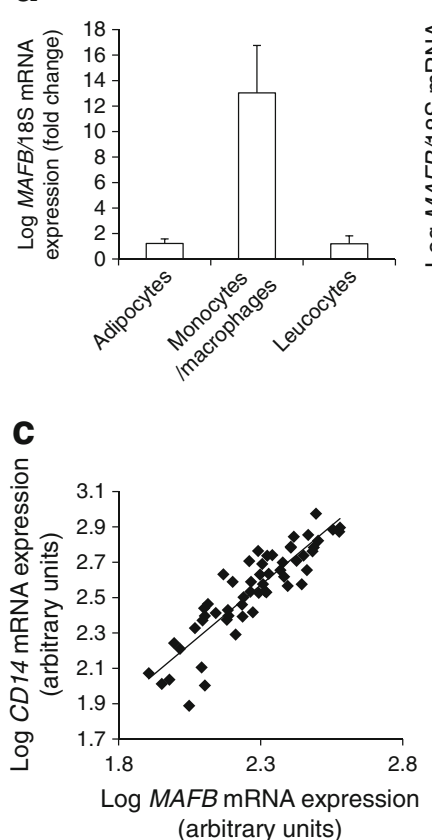

Fig. $6 M A F B$ is highly expressed in adipose-derived macrophages, and its expression correlates with macrophage markers and inflammation. (a) $M A F B$ expression was evaluated in FACS-sorted scWAT adipocytes, macrophages and leucocytes $(n=11-12)$ by qRT-PCR. (b) Comparison of $M A F B$ mRNA expression between isolated adipocytes and paired intact adipose tissue from seven lean (white bars) and six obese (black bars) individuals. $M A F B$ expression in lean adipocytes is used as a calibrator. Correlation of $M A F B$ with $C D 14$ mRNA detected by microarray (Pearson $r=0.8887, p<0.0001)(\mathbf{c})$ and adipose-secreted TNF- $\alpha$ (Pearson $r=$ $0.6458, p<0.0001)(\mathbf{d})$. TNF- $\alpha$ secretion was quantified using ELISA and is shown as ng per $10^{7}$ adipocytes. The number of cells was calculated based on TG content and the average cell volume $(n=56)$. Values have been logarithmically transformed $\left(\log _{10}\right)$ for normal distribution. $* * * p<0.001$

adipocytes as well as basal expression of CCL2 in primary in vitro differentiated human adipocytes. Thus, MAFB is a regulator of inflammation in adipocytes in a similar manner as is described for monocytes [19]. However, MAFB expression is in itself differentially affected by TNF- $\alpha$ in adipocytes vs macrophages. In addition, $M A F B$ expression and regulation patterns in human WAT suggest that MAFB is, in the context of obesity, primarily involved in regulation of the proinflammatory phenotype of macrophages within WAT and may drive development of insulin resistance.

MAFB has been reported to regulate differentiation of several cell types, including macrophages [15]. We show that $M A F B$ expression is highly induced by adipogenesis in human primary adipocytes. This could reflect the regulation of $M A F B$ by C/EBPS reported in keratinocytes [20]. Although MAFB does not drive adipogenesis per se, the strong upregulation of MAFB expression during differentiation implies a possible importance for e.g. metabolism or inflammation in the mature adipocyte.
Further, our data show that MAFB does not exert direct effects on adipocyte metabolism in vitro. However, MAFB knockdown attenuates the unfavourable effects caused by TNF- $\alpha$ on lipid accumulation and lipolysis. MAFB is also required for full effects of TNF- $\alpha$-mediated induction of IL- 6 expression. Therefore our findings indicate that MAFB potentiates TNF- $\alpha$-induced metabolically unfavourable biology in adipocytes. Interestingly, adipocytes may have developed a compensatory mechanism to downregulate MAFB in the proinflammatory environment. It is also likely that MAFB expression is under similar control to that of other adipocyte-specific genes such as PLIN1 and HSL, which are strongly induced by adipogenesis and downregulated by TNF- $\alpha$. Our data suggest that SPI1 could be involved in cell type-specific regulation of MAFB. However, at present we do not have strong experimental evidence on the mechanism of differential MAFB regulation in adipocytes vs THP-1 cells.

MAFB expression in human scWAT is mostly contributed by adipose tissue macrophages. Therefore the level of $M A F B$ mRNA correlates well with macrophage markers such as CD14 and CD68 (Fig. 6c and data not shown) and secretion of the proinflammatory cytokine TNF- $\alpha$ (Fig. 6d). Furthermore, $M A F B$ correlates negatively with favourable metabolic measures such as insulin and isoprenaline responsiveness (Fig. 2). These data suggest that MAFB is a marker of a proinflammatory status of WAT and most likely directly reflects the amount of macrophage infiltration. MAFB expression was found 12-fold higher in WAT-derived monocytes/ macrophages than in adipocytes. Thus, the increased MAFB expression originating from macrophage infiltration within obese WAT will, in the in vivo context, likely outweigh the attenuation of MAFB by TNF- $\alpha$ in adipocytes within the same tissue, in total causing increased $M A F B$ expression in obese WAT. It should however be noted that in morbidly obese patients, the expression of $M A F B$ was unexpectedly lower in omental compared with subcutaneous fat tissue - a depot generally considered to display a higher level of inflammation (ESM Fig. 4). The morbid obesity present in these patients may, hypothetically, cause extreme peripheral hypoxia, leading to a relative hyper-inflamed state in scWAT compared with omental tissue of the same individuals. Contrary to general belief, it has also been repeatedly reported that the level of TNF- $\alpha$ secretion is not higher in visceral than subcutaneous fat from lean nor obese individuals [25-27]. It would be desirable to investigate MAFB expression in paired samples of subcutaneous and omental fat of lean and overweight individuals, but such material is difficult to obtain due to ethical reasons. At present we do not know if MAFB expression is enriched in different macrophage subtypes such as M1 and M2 [28] and if MAFB is involved in macrophage polarisation. This question might be answered by deeper SVF cell sorting 
and transcriptome profiling of distinct scWAT macrophage populations in larger cohorts, which was beyond the scope of this study.

In summary, our findings suggest that MAFB positively regulates inflammation in both adipocytes and macrophages. Inflammation originating in adipose tissue has been shown to cause obesity-related insulin resistance [7], and therefore agents downregulating MAFB expression might have positive effects on adipose tissue metabolism and systemic glucose homeostasis. However, MAFB deficient macrophages have been shown to enter a continuous proliferation state without losing their macrophage phenotype [29]. Therefore, a full loss of MAFB could increase the amount of differentiated macrophages in adipose tissue - a feature that is generally considered metabolically disadvantageous. It remains to be established through which mechanisms differential regulation of MAFB is implicated in adipocytes vs macrophages. Meanwhile, $M A F B$ can be used as a model gene to study differential effects of TNF- $\alpha$ signalling in these two cell types, which are important for metabolic complications caused by obesity.

Taken together, we have identified MAFB as a novel regulator of human WAT inflammation and of TNF- $\alpha$-mediated effects on lipid accumulation and lipolysis. We have shown that MAFB is expressed in differentiated human adipocytes and regulates expression of inflammatory genes. However, MAFB can be named as a prominent macrophage marker in WAT and strongly reflects the WAT proinflammatory status in obesity and the metabolic syndrome.

Acknowledgements The technical assistance of G. Åström, E. Sjölin, E. Dungner, K. Wåhlén, B.-M. Leijonhufvud, Y. Widlund and K. Hertel (Department of Medicine Huddinge, Karolinska Institutet, Sweden) is greatly appreciated.

Funding This study was supported by the Swedish Research Council, NovoNordisk Foundation, Karolinska Institutet and Tore Nilsson Foundation.

Duality of interest The authors declare that there is no duality of interest associated with this manuscript.

Author contribution Conception and design was carried out by AMLP and JL; all authors were involved with the acquisition, analysis and interpretation of data; AMPL, JRA and JL drafted the article, while critical revising of the manuscript was carried out by $\mathrm{CB}, \mathrm{DL}, \mathrm{NV}, \mathrm{JK}$, BS, LB and PA. All authors approved the final version of the paper to be published. JL is the guarantor of this work.

\section{References}

1. Haslam DW, James WP (2005) Obesity. Lancet 366:1197-1209

2. Anderson EK, Gutierrez DA, Hasty AH (2010) Adipose tissue recruitment of leukocytes. Curr Opin Lipidol 21:172-177
3. Har D, Carey M, Hawkins M (2013) Coordinated regulation of adipose tissue macrophages by cellular and nutritional signals. J Investig Med 61:937-941

4. Fried SK, Bunkin DA, Greenberg AS (1998) Omental and subcutaneous adipose tissues of obese subjects release interleukin-6: depot difference and regulation by glucocorticoid. J Clin Endocrinol Metab 83:847-850

5. Sewter CP, Digby JE, Blows F, Prins J, O'Rahilly S (1999) Regulation of tumour necrosis factor-alpha release from human adipose tissue in vitro. J Endocrinol 163:33-38

6. Ouchi N, Parker JL, Lugus JJ, Walsh K (2011) Adipokines in inflammation and metabolic disease. Nat Rev Immunol 11:85-97

7. Wellen KE, Hotamisligil GS (2005) Inflammation, stress, and diabetes. J Clin Invest 115:1111-1119

8. Wang PW, Eisenbart JD, Cordes SP, Barsh GS, Stoffel M, Le Beau MM (1999) Human KRML (MAFB): cDNA cloning, genomic structure, and evaluation as a candidate tumor suppressor gene in myeloid leukemias. Genomics 59:275-281

9. Kataoka K, Fujiwara KT, Noda M, Nishizawa M (1994) MafB, a new Maf family transcription activator that can associate with Maf and Fos but not with Jun. Mol Cell Biol 14:7581-7591

10. Manzanares M, Trainor PA, Nonchev S et al (1999) The role of kreisler in segmentation during hindbrain development. Dev Biol 211:220-237

11. Sieweke MH, Tekotte H, Frampton J, Graf T (1996) MafB is an interaction partner and repressor of Ets-1 that inhibits erythroid differentiation. Cell 85:49-60

12. Zanocco-Marani T, Vignudelli T, Parenti S et al (2009) TFE3 transcription factor regulates the expression of MAFB during macrophage differentiation. Exp Cell Res 315:1798-1808

13. Smink JJ, Begay V, Schoenmaker T, Sterneck E, de Vries TJ, Leutz A (2009) Transcription factor C/EBPbeta isoform ratio regulates osteoclastogenesis through MafB. EMBO J 28:1769-1781

14. Artner I, Blanchi B, Raum JC et al (2007) MafB is required for islet beta cell maturation. Proc Natl Acad Sci U S A 104:3853-3858

15. Kelly LM, Englmeier U, Lafon I, Sieweke MH, Graf T (2000) MafB is an inducer of monocytic differentiation. EMBO J 19: 1987-1997

16. Matsuoka TA, Zhao L, Artner I et al (2003) Members of the large Maf transcription family regulate insulin gene transcription in islet beta cells. Mol Cell Biol 23:6049-6062

17. Artner I, Le Lay J, Hang Y et al (2006) MafB: an activator of the glucagon gene expressed in developing islet alpha- and beta-cells. Diabetes 55:297-304

18. Guo S, Dai C, Guo M et al (2013) Inactivation of specific beta cell transcription factors in type 2 diabetes. J Clin Invest 123: 3305-3316

19. Zhang Y, Chen Q, Ross AC (2012) Retinoic acid and tumor necrosis factor-alpha induced monocytic cell gene expression is regulated in part by induction of transcription factor MafB. Exp Cell Res 318: 2407-2416

20. Borrelli S, Fanoni D, Dolfini D et al (2010) C/EBPdelta gene targets in human keratinocytes. PLoS One 5, e13789

21. Gao H, Mejhert N, Fretz JA et al (2014) Early B cell factor 1 regulates adipocyte morphology and lipolysis in white adipose tissue. Cell Metab 19:981-992

22. Pettersson AM, Stenson BM, Lorente-Cebrian S et al (2013) LXR is a negative regulator of glucose uptake in human adipocytes. Diabetologia 56:2044-2054

23. Pettersson AT, Mejhert N, Jernas M et al (2011) Twist1 in human white adipose tissue and obesity. J Clin Endocrinol Metab 96: 133-141

24. Cheng P, Chen C, He HB et al (2013) miR-148a regulates osteoclastogenesis by targeting V-maf musculoaponeurotic fibrosarcoma oncogene homolog B. J Bone Miner Res 28:1180-1190 
25. van Harmelen V, Dicker A, Ryden M et al (2002) Increased lipolysis and decreased leptin production by human omental as compared with subcutaneous preadipocytes. Diabetes 51: 2029-2036

26. Dusserre E, Moulin P, Vidal H (2000) Differences in mRNA expression of the proteins secreted by the adipocytes in human subcutaneous and visceral adipose tissues. Biochim Biophys Acta 1500:88-96
27. Montague CT, Prins JB, Sanders L et al (1998) Depot-related gene expression in human subcutaneous and omental adipocytes. Diabetes 47:1384-1391

28. Martinez FO, Gordon S (2014) The M1 and M2 paradigm of macrophage activation: time for reassessment. F1000Prime Rep 6:13

29. Aziz A, Soucie E, Sarrazin S, Sieweke MH (2009) MafB/c-Maf deficiency enables self-renewal of differentiated functional macrophages. Science 326:867-871 